\title{
The Simulation Teaching Method for the Routing Protocol Content of Computer Network Course
}

\author{
Henghua Shi ${ }^{1, \text { a }}$, Renlong Zhang ${ }^{1, b^{*}}$ and Chengkui Guo ${ }^{1, c}$ \\ ${ }^{1}$ School of Computer and Information Engineering, Beijing University of Agriculture, Beijing, China \\ ahenghuashi@163.com, ${ }^{b}$ zrl@bac.edu.cn, ${ }^{c}$ gck@bac.edu.cn \\ *The corresponding author
}

Keywords: Simulation; Teaching Method; Routing Protocol; Computer Network; RIP

\begin{abstract}
The routing protocol is the core of the communication technology is one of the important knowledge points in the teaching of computer network course. In the traditional teaching experiment, we need to purchase a lot of router equipments for meeting the experimental hardware requirements. We present a simulation teaching method based on Packet Tracer simulation technology to construct network topology and simulate the routing protocol such as RIP. The simulation results show that compared with the actual network hardware equipment, the simulation teaching method can more easily and effectively configure the network topology to learn the routing protocol content in the computer network course.
\end{abstract}

Acknowledgement: Corresponding author is Zhang Renlong. The authors would like to acknowledge the supports provided by 2016 General Scientific Research Project of Beijing Municipal Education Commission (PXM2016_014207_000008).

\section{计算机网络课程中网络路由协议内容的仿真教学}

\section{方法}

\author{
石恒华 ${ }^{1}$ ，张仁龙 ${ }^{1}$ ，郭成鬼 ${ }^{1}$ \\ 1 北京农学院计算机与信息工程学院，北京中国
}

摘要: 网络路由协议是计算机网络的一个重要知识点。在传统的教学中需要购置大量的路由器设备, 才能满足相关实验 的硬件要求。提出一个基于 Packet Tracer 仿真技术，构建网络拓扑结构，模拟虚拟 RIP 为代表的网络路由协议的仿真教学 方法。仿真结果表明，与实际网络硬件设备相比，该仿真教学方法可以更容易、更有效地配置网络拓扑来学习计算机网络课 程中网络路由协议内容。

关键词: 仿真；教学方法；路由协议；计算机网络；路由信息协议

\section{1 前言}

计算机网络最重要的技术是网络通信连接技术，越来越多的高校在计算机网络课程中开展网络路由协 议内容的教学。进行网络路由协议的教学需要许多实际网络设备, 如路由器等。但是, 许多高校对网络实 验缺乏足够的实际网络设备。

在对教育类网络仿真技术研究 [1] [2]的基础上，选择 Packet Tracer 仿真软件进行计算机网络课程中 网络路由协议内容的仿真教学。应用教育类网络仿真技术, 通过对仿真界面直观的观察和直接的互动, 学 生可以更容易理解相关的理论概念、更好地拓展思维空间 [3]。本文基于 Packet Tracer 仿真软件构建一 个网络拓扑结构, 模拟以 RIP 为例的网络路由协议内容的仿真教学。实验结果表明, 与实际网络硬件设备 相比, 基于 Packet Tracer 的仿真教学方法可以更容易、更有效地配置网络拓扑来学习计算机网络课程中 
网络路由协议内容。

\section{Packet Tracer仿真技术}

Packet Tracer [4]是一个教育类的网络通信仿真软件。与其他仿真软件, 如 Opnet [5]、NS2 [6]、GNS3 [7] 等相比, Packet Tracer 是思科公司开发的最流行和最简单的仿真工具, 包括各种高校教学网络技术与产 品, 能够一定程度上替代物理网络教学设备。在课堂上允许学生创建一个几乎无限网络设备数量的网络, 鼓励实践、发现并排除故障。基于模拟的学习环境帮助学生发展二十一世纪的技能, 提升决策能力, 培养 创造性和批判性思维, 以及解决现实网络问题 [8]。

\section{3 网络路由协议}

路由协议指定路由器如何相互通信, 并允许传播信息来选择任意两个节点之间的路由。路由协议首先 在相邻的邻居之间共享路由器的路由表信息, 然后在整个网络中共享路由表信息。通过这种方式, 路由器 获得整个网络拓扑结构的知识。

有许多类型的网络路由协议, 主要分为以下两大类: 内部网关协议 (IGP) 和外部网关协议 (EGP)。 内部网关协议（IGP） 又分为：距离矢量路由协议，如路由信息协议（RIP） [9]; 链路状态协议, 如开放 最短路径优先路由协议 (OSPF) [10]。外部网关协议（EGP）是 Internet 上使用自治系统之间交换路由信 息的路由协议, 如边界网关协议 (BGP)。在所有路由协议中, RIP 是最简单且易于配置的路由协议之一。

在当前的网络环境，与 RIP 的收玫时间相比，EIGRP、OSPF 协议的扩展性较差。等跳等因素的限制， 使得 RIP 只适合在小规模网络中应用。因此, RIP 不是路由的首选方案, 但是, RIP 很容易配置, 且不像 其他协议, RIP 不需要路由器上的任何参数。为此, 以 RIP 为例进行网络路由协议内容的教学是一个较为 简单易学的方式。基于 Packet Tracer 仿真软件构建一个网络拓扑结构, 模拟以 RIP 为例的网络路由协议, 进行网络路由协议仿真教学方法研究。

\section{4 仿真教学方法}

\section{1 仿真实验配置}

基于 Packet Tracer 仿真软件建一个网络拓扑结构, 包括两个路由器和四个 PC, 并配置 RIP 网络路由 协议, 如图 1 所示。

在模拟网络拓扑, Router 0 的 $\mathrm{Fa} 0 / 0$ 和 $\mathrm{Fa} 0 / 1$ 接口分别与 PCO 和 PC1 连接, Router 1 的 $\mathrm{Fa} 0 / 0$ 和 $\mathrm{Fa} 0 / 1$ 接口分别与 PC2 和 PC3 连接, Router0 的 S0/0/0 与 Router 1 的 S0/0/0 连接。所有路由器和 PC 的 IP 地址

等配置信息, 如表 1 所示。

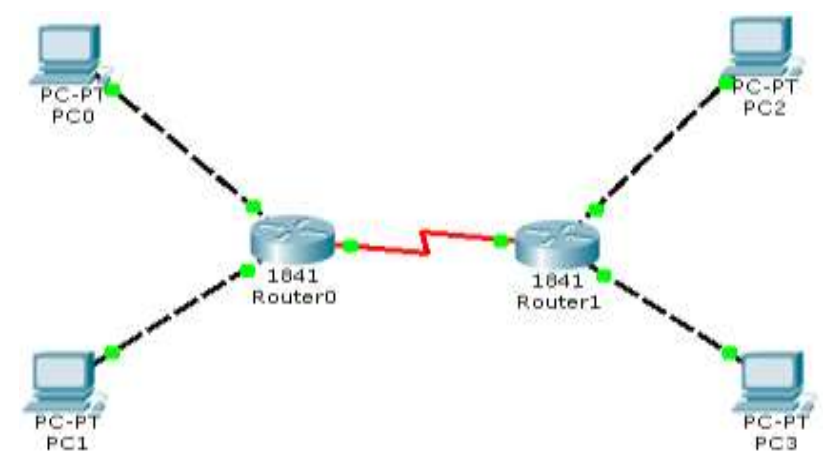

图 1 网络拓扑结构图 


\section{2 仿真结果与分析}

图 1 中, 从 PC0 和/或 PC1, 向 PC2 和/或 PC3 的所有数据包, 需要通过对 Router0 和 Router1。例如, 从 PC0 到 PC3 进行 ping 命令。所有的 ping 命令的以太网帧, 在 Router0 上被转换成 HDLC 帧, 如图 2 所 示。同理, 这些 ping 命令的 HDLC 帧, 再在 Router1 上被转换回以太网帧，如图 3 所示。

表 1 所有路由器和 PC 的 IP 地址等配置信息

\begin{tabular}{lllll}
\hline 设备 & 接口 & IP 地址 & 子网掩码 & 默认网关 \\
\hline PC0 & NIC & 192.168 .1 .1 & 255.255 .255 .0 & 192.168 .1 .254 \\
PC1 & NIC & 192.168 .2 .1 & 255.255 .255 .0 & 192.168 .2 .254 \\
PC2 & NIC & 192.168 .3 .1 & 255.255 .255 .0 & 192.168 .3 .254 \\
PC3 & NIC & 192.168 .4 .1 & 255.255 .255 .0 & 192.168 .4 .254 \\
Router0 & Fa0/0 & 192.168 .1 .254 & 255.255 .255 .0 & N/A \\
& Fa0/1 & 192.168 .2 .254 & 255.255 .255 .0 & N/A \\
& S0/0/0 & 192.168 .5 .1 & 255.255 .255 .0 & N/A \\
Router1 & $\mathrm{Fa} 0 / 0$ & 192.168 .3 .254 & 255.255 .255 .0 & N/A \\
& $\mathrm{Fa} 0 / 1$ & 192.168 .4 .254 & 255.255 .255 .0 & $\mathrm{~N} / \mathrm{A}$ \\
& $\mathrm{S} 0 / 0 / 0$ & 192.168 .5 .2 & 255.255 .255 .0 & $\mathrm{~N} / \mathrm{A}$ \\
\hline
\end{tabular}

当所有的数据包, 从 PC0 到 Router0 时, Router0 将以太网帧中得到目的 IP 地址 192. 168. 4. 1, 并在 Router0 的路由表中找出正确的路由条目, 对外接口为 serial0/0/0。然后, 所有的数据包从 router 0 的 到 serial0/0/0 接口被转发出去。因为, Router0 到 Router 1 之间是 HDLC 链路, 所有的数据包都被 Router0 转换成 HDLC 帧。Router1 将收到 Router0 转发出的 HDLC 帧转换成以太网帧, 然后, 所有的数据包应该发 送到 PC3。
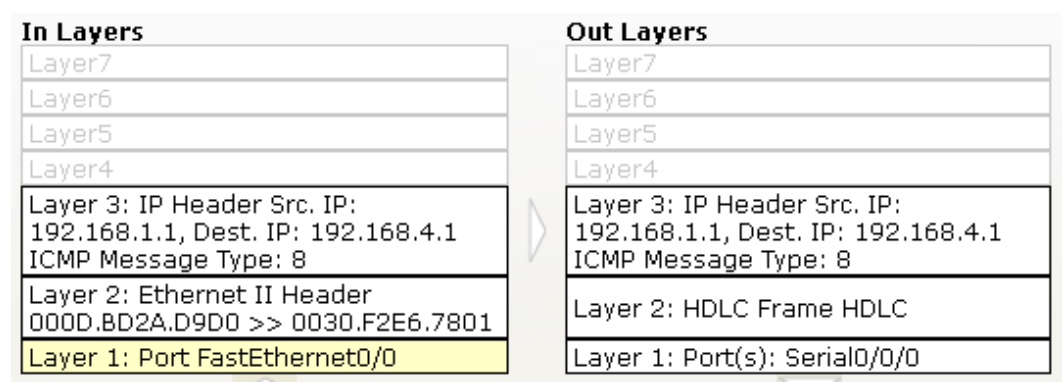

图 2 Router 0 上的数据帧格式转换
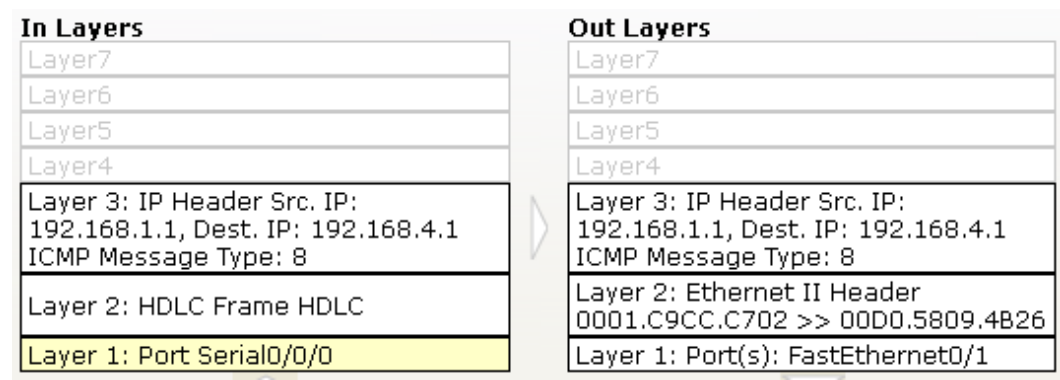

图 3 Router 1 上的数据帧格式转换

在这个过程中, 最为重要的是 Router0 的路由表, 和如何找出正确的路由条目。本仿真教学方法中, 
在 Router 0 和 Router 1 上配置的网络路由协议是 RIP 协议，相关信息如下:

Router0\#

C 192.168.1.0/24 is directly connected, FastEthernet0/0

C 192.168.2.0/24 is directly connected, FastEthernet0/1

R 192.168.3.0/24[120/1] via 192.168.5.2, 00:00:10, Serial0/0/0

R 192.168.4.0/24[120/1] via 192.168.5.2, 00:00:10, Serial0/0/0

C 192.168.5.0/24 is directly connected, Seria10/0/0

Router1\#

R 192.168.1.0/24[120/1] via 192.168.5.1, 00:00:10, Serial0/0/0

R 192.168.2.0/24[120/1] via 192.168.5.1, 00:00:10, Serial0/0/0

C 192.168.3.0/24 is directly connected, FastEthernet0/0

C 192.168.4.0/24 is directly connected, FastEthernet0/1

C 192.168.5.0/24 is directly connected, Seria10/0/0

\section{5 结论}

随着互联网的不断发展，计算机网络技术也被应用到越来越广泛的生物信息等高性能计算领域中。网 络路由协议作为计算机网络技术的核心内容是计算机网络课程教学的重点和难点。为了有效的进行网络路 由协议的教学, 通常需要购置大量的路由器设备, 在缺乏相关网络设备时很难开展相关的教学实验。

在对教育类网络仿真技术研究的基础上，基于 Packet Tracer 仿真软件构建一个网络拓扑结构，模拟 以 RIP 为例的网络路由协议内容的仿真教学。实验结果表明, 与实际网络硬件设备相比, 基于 Packet Tracer 的仿真教学方法能够方便的完成 RIP 等网络路由协议的配置, 并进行相关的仿真实验教学工作。可以更容 易、更有效地配置网络拓扑来学习计算机网络课程中网络路由协议内容。

\section{6 致谢}

本文研究得到通讯作者张仁龙老师的大力帮助, 本文为 2016 年北京市教委科研计划一般项目 (PXM2016_014207_000008) 的阶段性成果之一。

\section{参考文献:}

[1] 谢伟增，金振乾．基于Packet Tracer 的《计算机网络技术》课程虚拟仿真实验教学. 信息与电脑，2017，4： 223-226.

[2] 范业仙，冯玮. 仿真技术在计算机网络课堂教学中的应用. 韶关学院学报, 2013，34(8)：24-27.

[3] Eric Gamess, Jesús Urbáez, Arturo Palacios. A Visual Teaching and Learning Environment for the Open Shortest Path First Routing Protocol. Ninth Latin American and Caribbean Conference for Engineering and Technology. 2011:1-10.

[4] Information on https://www. netacad. com/web/about-us/cisco-packet-tracer.

[5] 陈敏. OPNET网络仿真. 清华大学出版社，北京，2015，2-5.

[6] Information on https://www. isi. edu/nsnam/ns/

[7] Information on http://www. gns3. net/documentation/ 
[8] Petcu D. , Iancu B., Peculea A., Dadarlat V., Cebuc E. Integrating Cisco Packet Tracer with Moodle platform: Support for teaching and automatic evaluation. 12th RoEduNet International Conference: Networking in Education and Research, RoEduNet 2013.

[9] Malkin, Gary Scott (2000). RIP: An Intra-Domain Routing Protocol. Addison-Wesley Longman. ISBN $\quad 0-201-43320-6$.

[10] Moy, John T. OSPF: Anatomy of an Internet Routing Protocol. Addison-Wesley. ISBN 978-0201634723.

[11] Albrightson, R., Garcia-Luna-Aceves, J. J. , \& Boyle, J. (1994, May). EIGRP a fast routing protocol based on distance vectors. In Proc. Networld/Interop, Vol. 94, p. 136-147.

\section{References}

[1] Xie Wei-zeng, Jin Zhen-qian. Virtual Simulation Experiment Teaching of Computer Network Technology based on Packet Tracer. China Computer\&Communication, 2017, Vol 4, p223-226.

[2] Fan Ye-wei, Feng Wei. Application of simulation technology in the class teaching of computer network. Journal of Shaoguan University, 2013, Vol 34(8), p24-27.

[3] Eric Gamess, Jesús Urbáez, Arturo Palacios. A Visual Teaching and Learning Environment for the Open Shortest Path First Routing Protocol. Ninth Latin American and Caribbean Conference for Engineering and Technology.2011:1-10.

[4] on https://www.netacad.com/web/about-us/cisco-packet-tracer.

[5] Chen M. OPNET Network Simulation. TingHua University Press, Beijing (2004), p.2-5. [6] Information on https://www.isi.edu/nsnam/ns/

[7] Information on http://www.gns3.net/documentation/

[8] Petcu D., Iancu B., Peculea A., Dadarlat V., Cebuc E. Integrating Cisco Packet Tracer with Moodle platform: Support for teaching and automatic evaluation. 12th RoEduNet International Conference: Networking in Education and Research, RoEduNet 2013.

[9] Malkin, Gary Scott (2000). RIP: An Intra-Domain Routing Protocol. Addison-Wesley Longman. ISBN 0-201-43320-6.

[10] Moy, John T. OSPF: Anatomy of an Internet Routing Protocol. Addison-Wesley. ISBN 978-0201634723.

[11] Albrightson, R., Garcia-Luna-Aceves, J. J., \& Boyle, J. (1994, May). EIGRP a fast routing protocol based on distance vectors. In Proc. Networld/Interop, Vol. 94, p.136-147. 